\title{
Precision medicine in cholangiocarcinoma
}

\author{
Antonio Pellino ${ }^{1}$, Fotios Loupakis ${ }^{2}$, Massimiliano Cadamuro ${ }^{3}$, Vincenzo Dadduzio ${ }^{2}$, Matteo Fassan $^{4}$, \\ Maria Guido ${ }^{4}$, Umberto Cillo ${ }^{1}$, Stefano Indraccolo ${ }^{5}$, Luca Fabris ${ }^{3,6}$ \\ ${ }^{1}$ Department of Surgery, Oncology and Gastroenterology (DISCOG), University of Padua, Padua, Italy; ${ }^{2}$ Medical Oncology Unit 1 , Clinical and \\ Experimental Oncology Department, Veneto Institute of Oncology IOV-IRCCS, Padua, Italy; ${ }^{3}$ Department of Molecular Medicine (DMM), \\ University of Padua, Padua, Italy; ${ }^{4}$ Department of Medicine (DIMED), Surgical Pathology Unit, University of Padua, Padua, Italy; ${ }^{5}$ Immunology \\ and Molecular Oncology Unit, Veneto Institute of Oncology IOV-IRCCS, Padua, Italy; ${ }^{6}$ Department of Internal Medicine, Yale Liver Center (YLC), \\ School of Medicine, Yale University New Haven, CT, USA \\ Contributions: (I) Conception and design: A Pellino, F Loupakis, M Cadamuro, L Fabris; (II) Administrative support: M Cadamuro; (III) Provision of \\ study materials or patients: A Pellino, F Loupakis, V Dadduzio, L Fabris; (IV) Collection and assembly of data: A Pellino, F Loupakis, M Cadamuro, \\ L Fabris; (V) Data analysis and interpretation: All authors; (VI) Manuscript writing: All authors; (VII) Final approval of manuscript: All authors. \\ Correspondence to: Luca Fabris, MD, PhD. Associate Professor of Gastroenterology, Department of Molecular Medicine, University of Padua, Viale G. \\ Colombo 3, Padua 35131, Italy. Email: luca.fabris@unipd.it.
}

\begin{abstract}
Cholangiocarcinoma is one of the epithelial cancers with the poorest prognosis and the narrowest therapeutic choice in humans. Compared with other cancer types, cholangiocarcinoma has been often neglected by oncology and liver research studies, thereby leaving many issues unsolved. Apart from the early and marked aggressiveness, one of the main reasons of the still unsatisfying clinical management of cholangiocarcinoma is its wide tumor heterogeneity needing more than other diseases a 'precision medicine' approach. In this regard, in the last few years there has been an awakening of interest aimed at dissecting the complex molecular and genomic profile of cholangiocarcinoma. Thus, a range of molecular players have been recently identified as putative mechanistic determinants of cholangiocarcinoma invasiveness, encompassing tyrosine kinase receptors, metabolic enzymes, transcription factors, small GTPases, ubiquitin ligases, and chromatin-remodelling proteins, whose aberrant expression may derive from stochastic mutations as well as from pro-oncogenic paracrine signals released by the stromal microenvironment, which is particularly exuberant in cholangiocarcinoma. Herein, we sought to overview the most relevant observations unravelling the genomic landscape of cholangiocarcinoma, and the prognostic and predictive biomarkers that consequently have been emerging. Then, we will discuss innovative treatment approaches derived from conventional chemotherapy, targeted therapies, antiangiogenic therapies and immunotherapy, and how they are opening new avenues towards a precision medicine in cholangiocarcinoma.
\end{abstract}

Keywords: Biliary tract cancer; gene mutations; tumor biomarker; stromal microenvironment

Received: 15 June 2018; Accepted: 03 July 2018; Published: 12 July 2018.

doi: $10.21037 / \operatorname{tgh} .2018 .07 .02$

View this article at: http://dx.doi.org/10.21037/tgh.2018.07.02

\section{Introduction}

Cholangiocarcinoma (CCA) is a primary liver cancer with features of cholangiocyte differentiation, the epithelial cell lining the intra and extrahepatic portions of the biliary tree. Classically, CCA has been always regarded as a 'rare' tumor, at least in Western countries, but over the last 15 years its incidence has steadily increased worldwide, and nowadays, it represents the second most common type of primary malignancy in the liver (15-20\% of cases) after hepatocellular carcinoma. Compared with hepatocellular carcinoma, CCA frequently develops on a background of non-cirrhotic liver, thus evading surveillance programs, and association with known risk factors is much less defined. However, chronic inflammation and cholestasis are conditions with increased risk of CCA development, 
including infection with hepatobiliary flukes (Opisthorchis viverrini), hepatolithiasis, primary sclerosing cholangitis, and congenital malformations of the bile ducts (e.g., Caroli's disease, choledochal cysts). In addition, chronic viral infections from hepatitis $\mathrm{B}$ and $\mathrm{C}$ virus ( $\mathrm{HBV}, \mathrm{HCV}$ ), as well as chronic liver disease associated with metabolic syndrome (non-alcoholic steatohepatitis), are emerging as new risk factors of CCA (1-3).

Notably, CCA is not only rare on epidemiological grounds, but also in terms of devoted studies, with a series of questions about pathogenesis, diagnosis and treatment left unanswered. Moreover, a major limitation of studies dealing with CCA is the 'rough' approach to evaluate CCA without bearing in mind the wide clinical heterogeneity related to the primary location within the biliary system. In colorectal cancer, clinical management improved markedly after recognizing the distinctive features of right-sided $v s$. left-sided tumors $(4,5)$. Following this example, by considering the merging point of the bile ducts of second order as the boundary line, CCA can be categorized into three different forms, intrahepatic cholangiocarcinoma (iCCA), perihilar cholangiocarcinoma (pCCA), and distal cholangiocarcinoma (dCCA), each with its own pathogenesis and clinical outcome (6). Most of clinical trials performed so far have considered iCCA, pCCA and dCCA as a whole group of diseases, sometimes including even gallbladder cancer, and this approach has hindered the development of the optimal therapy aimed at the specific type of biliary tract cancer. Thus, only small improvements in the management of CCA have been achieved and prognosis has remained poor with a median survival below 2 years and a survival rate of less than $10 \%$ owed to the early and strong invasiveness of the tumor.

The discovery of new oncogenic drivers is a pre-requisite for the development of innovative strategies and for the identification of biomarkers predicting the response to therapy. In recent years, cutting-edge technologies such as DNA and/or RNA sequencing, or fluorescence in situ hybridization (FISH) coupled with the more conventional immunohistochemistry (IHC) have contributed to unravel the complex genomic and transcriptomic landscape of CCA. Studies performed at different somatic, epigenetic, proteomic and microRNA levels have shed light on the intricate molecular mechanisms underlying pathogenesis of CCA in relation to the specific subtype. In this review, we will first outline the emerging concepts on the genomic profiling of CCA. Prognostic and predictive biomarkers will be discussed afterwards, before analyzing the new therapeutic avenues aimed at a precision medicine that are emerging.

\section{The evolving genomic landscape in CCA}

A number of genetic alterations, including point mutations, copy number variations and gene fusions are progressively emerging in CCA, perturbing fundamental cell functions, such as DNA repair, cell cycle regulation, receptor tyrosine kinase signaling, and epigenetic regulation of gene expression (Table 1).

In a comprehensive analysis published in 2015 by Nakamura et al., the molecular profile of 260 Japanese patients with biliary tract cancer (145 iCCA, 86 pCCA/ dCCA and 29 gallbladder cancers) was characterized by next generation sequencing (NGS) reporting a high rate $(38.9 \%)$ of potentially actionable genetic alterations diverging across the different anatomical subtypes (7). The fibroblastic growth factor (FGF) pathway is involved in several biological processes including angiogenesis, cell proliferation, migration, differentiation and wound healing. A deregulation of FGF signaling has been described in a number of epithelial cancers including CCA. In particular, a variety of FGFR2 rearrangements with gene fusion have been found in about $10 \%$ to $16 \%$ of iCCA, but much rarer in pCCA/dCCA $(8,9)$. A higher rate of FGFR2 fusions (45\%) was reported by Sia et al. by RNA and exome-sequencing analyses in a cohort of 107 resected iCCA patients, resulting the most frequent targetable alteration. A number of studies have recently reported new FGFR2 fusions (FGFR2-KCTD1, FGFR2-TXLNA, FGFR2-PPHLN1) underlining further the importance of this pathway in the carcinogenesis of $\operatorname{iCCA}(7,10)$.

On the other hand, fusion genes involving PRKACA and $P R K A C B$, both members of the serin/threonine protein kinase and catalytic subunits of the protein kinase $\mathrm{A}$, have been identified solely in $\mathrm{pCCA} / \mathrm{dCCA}(7)$.

Rearrangements of the proto-oncogene ROS1 have been found in a large variety of malignancies, including non-small cell lung carcinoma (NSCLC), glioblastoma multiforme, gastric and colon carcinomas. These rearrangements result in a fusion protein harboring a constitutive activation of the ROS1 kinase domain that promotes oncogenesis. ROS1 gene fusions have been reported in the $8-9 \%$ of CCAs $(7,11)$, and in a recent work, FIG-ROS1 rearrangement was found in $4 / 25 \mathrm{pCCA} / \mathrm{dCCA}$, while in none out of 26 iCCA (12).

Genomic studies have highlighted the concept that organ-specific alterations in epigenetic regulators are common in CCA. Noteworthy, Jiao et al. have discovered 
Table 1 Genetic variants in cholangiocarcinoma (CCA)

\begin{tabular}{|c|c|c|c|}
\hline Gene & Prevalence in CCA & Gene significance & Other tumors with known gene involvement* \\
\hline $\begin{array}{l}\text { PRKACA/PRKACB } \\
\text { rearrangements }\end{array}$ & Rare $(\mathrm{pCCA} / \mathrm{dCCA})$ & $\begin{array}{l}\text { Member of the protein kinase } \mathrm{C} \text { family is } \\
\text { involved in several cellular processes such } \\
\text { as cell cycle, adhesion, transformation, } \\
\text { angiogenesis and aging }\end{array}$ & $\begin{array}{l}\text { Breast, lung, skin, stomach, and thyroid } \\
\text { cancer }\end{array}$ \\
\hline $\begin{array}{l}\text { ROS1 } \\
\text { rearrangements }\end{array}$ & $8-9 \%$ & $\begin{array}{l}\text { Proto-oncogene encoding a membrane } \\
\text { protein with tyrosine kinase activity }\end{array}$ & $\begin{array}{l}\text { Ovarian cancer and sarcoma, glioblastoma, } \\
\text { NSCLC, }\end{array}$ \\
\hline IDH1/IDH2 mutations & $25 \%$ (iCCA) & $\begin{array}{l}\text { Metabolic enzymes involved in oxidative } \\
\text { stress, glutathione metabolism, and citric } \\
\text { acid cycle; IDH defects induce accumulation } \\
\text { of (D)-2-hydroxyglutarate }\end{array}$ & $\begin{array}{l}\text { Acute lymphocytic and myeloid leukemia, } \\
\text { astrocytoma, bone cancers, and brain stem } \\
\text { glioma }\end{array}$ \\
\hline HER2 amplification & $\begin{array}{c}11-20 \%(\mathrm{pCCA} \\
\mathrm{dCCA})\end{array}$ & $\begin{array}{l}\text { Tyrosine kinase receptor belonging to the EGF } \\
\text { receptor family, regulating cell morphogenesis, } \\
\text { development, and proliferation }\end{array}$ & $\begin{array}{l}\text { Bladder, breast, lung, ovarian, gastric, } \\
\text { colorectal and prostate cancers, } \\
\text { medulloblastoma, and osteosarcoma }\end{array}$ \\
\hline TP53 mutation & $\begin{array}{l}27 \%(\mathrm{iCCA}), 40 \% \\
\quad(\mathrm{pCCA} / \mathrm{dCCA})\end{array}$ & $\begin{array}{l}\text { Tumor suppressor gene involved in cell cycle } \\
\text { arrest, cell apoptosis, cell senescence, and } \\
\text { DNA repair }\end{array}$ & $\begin{array}{l}\text { Adrenocortical, bladder, breast, colorectal, } \\
\text { endometrial, lung, esophageal, prostate, and } \\
\text { pancreatic cancers, hepatocellular carcinoma, } \\
\text { leukemia, melanoma, medulloblastoma, } \\
\text { NSCLC, and osteosarcoma }\end{array}$ \\
\hline PTEN mutation & $4 \%$ & $\begin{array}{l}\text { Tumor suppressor gene preventing cell } \\
\text { proliferation }\end{array}$ & $\begin{array}{l}\text { Breast, endometrial, lung, prostate, skin } \\
\text { and gastric cancers, astrocytoma, and } \\
\text { glioblastoma }\end{array}$ \\
\hline CDKN2A mutation & $47 \%(\mathrm{iCCA})$ & $\begin{array}{l}\text { Tumor suppressor gene inhibiting cell cycle } \\
\text { and proliferation }\end{array}$ & $\begin{array}{l}\text { Adrenocortical, bladder, breast, colorectal, } \\
\text { head and neck, lung, prostate, and pancreatic } \\
\text { cancers, Hodgkin lymphoma, osteosarcoma, } \\
\text { melanoma, rhabdomyosarcoma }\end{array}$ \\
\hline MET amplification & $2 \%$ (iCCA) & $\begin{array}{l}\text { Proto-oncogene member of the tyrosine } \\
\text { kinase receptor family }\end{array}$ & $\begin{array}{l}\text { Breast, cervical, colorectal, esophageal, } \\
\text { prostate, and gastric cancers, NSCLC, papillary } \\
\text { renal cell carcinoma, hepatocellular carcinoma, } \\
\text { and various head and neck cancers }\end{array}$ \\
\hline
\end{tabular}

${ }^{*}$, only cancers with $>5$ original articles published on PubMed citing the genetic variant have been included. 
inactivating mutations in multiple chromatin-remodeling genes in at least a half of iCCA (13). Genes encoding isocitrate dehydrogenase (IDH1, IDH2), are among the most common genetic lesions in iCCA (25\%) and they lead to an accumulation of 2-hydroxyglutarate (2-HG), an oncometabolite inhibiting members of the family of $\alpha$-ketoglutarate-dependent dioxygenase enzymes, resulting in epigenetic and genetic dysregulation and oncogenesis (14). Interestingly FGFR2 rearrangements and IDH1 mutations seem to be mutually exclusive (15). In addition to IDH1 and $I D H 2$, mutations in other genes involved in the assembly of chromatin structure, such as MLL2 and MLL3 and components of the switch/sucrose non fermentable (SWI/ SNF) nucleosome-remodelling complex (including ARID1A, ARID1B, ARID2, BAP1, PBRM1, SMARCA2, SMARCA4 and SMARCAD1) are more frequent in iCCA (13).

Both amplifications and mutations in the epidermal growth factor receptor (EGFR) gene have been found in CCA. EGFR overexpression occurs in $16 \%$ of CCAs, with a slight prevalence in iCCA (11-27\%) compared with pCCA/dCCA $(5-19 \%)(16,17)$. On the other hand, EGFR mutations have been reported in the $10-15 \%$ of CCA, but without a clear predominance between iCCA and pCCA/ dCCA (18-20). Human epidermal growth factor 2 (HER 2) amplification has been reported in up to $11-20 \%$ of pCCA/ dCCA while is rare in iCCA (21).

Alterations of the TP53 gene affecting DNA repair mechanisms, which are commonly present in gallbladder cancer $(59 \%)$, are also frequent in CCA, being more prevalent in pCCA/dCCA (40\%) than in iCCA (27\%) (22). The RAS/RAF/MEK and the mitogen-activated protein kinase/extracellular signal-regulated kinase (MAPK/ERK) pathway is defective in a large number of epithelial tumors including CCAs. Mutations of the proto-oncogene KRAS are frequently reported in CCA and have been detected in $22 \%$ of iCCA and in $42 \%$ of pCCA/dCCA, whilst $B R A F$ (1-5\%), PIK3CA (4-9\%), and PTEN (4\%) mutations are uncommon in all the subtypes $(22,23)$.

Focal losses of the cyclin dependent kinase inhibitor 2A $(C D K N 2 A)$, a gene encoding proteins (p14 and p16) involved in the negative regulation of the cell cycle, have been recently reported in a significant proportion (47\%) of iCCA (15). In contrast, MET amplification has been rarely identified in iCCA (2\%), and not in pCCA/dCCA (22).

\section{Genomic profiling and relationship with risk factors}

Whether specific patterns of somatic mutations are related to the different risk factors of CCA is uncertain. A whole exome sequencing analysis performed in 108 cases of CCAs identified different mutational patterns between forms caused by liver fluke infestations with $\mathrm{O}$. viverrini and non-liver fluke-related tumours. BAP1, IDH1 and $I D H 2$ mutations were more common in non-O. viverrini CCAs, while TP53, KRAS, MLL3, ROBO2, RNF43 and PEG3 had major mutation rate in $\mathrm{O}$. viverrini-related CCAs (24).

Furthermore, a different mutation profile was reported in iCCAs with chronic advanced liver disease (higher for EGFR and lower for KRAS, MLH1, GNAS) compared with iCCAs arising in normal liver, where mutations in PIK3CA, PTEN, CDKN2A, and TP53 were detected (25).

Jusakul et al. (26) have recently analyzed 489 CCAs from 10 countries using integrated genomic, epigenomic and transcriptomic analysis categorizing four CCA clusters: clusters 1-2 (fluke positive) enriched in TP53 mutations and ERBB2 amplifications; clusters 3-4 (fluke negative) characterized by high number of epigenomic mutations (IDH1, IDH2, BAP1), FGFR/PRKA gene rearrangements and PD-1/PD-L2 expression. Of note, different patterns of DNA hypermethylation can be observed between cluster 1 and 4 . Altogether, these findings suggest that different environmental, epigenetic and genetic factors cooperate to generate distinct molecular signatures in CCA.

\section{Prognostic and predictive factors}

In previous works, classical biochemical and histopathological signs, such as low preoperative bilirubin, clean resection margins (R0) and well-differentiated tumor histology were associated to a better prognosis for CCA. Conversely, less-differentiated grading, involvement of surgical margins (R1), perineural involvement, vascular or lymphatic invasion was related to a worst survival (27). Among genetic mutations, some implications with CCA outcome have been recently reported. FGFR2 gene fusion was associated with a relatively indolent disease course and with a significant survival advantage (28). However, these data were not confirmed in an Asian cohort study, where FGFR2 rearrangements were not related to an improved overall survival (OS). CCAs harboring KRAS mutations were characterized by a major involvement of adjacent organs and by R1 margin status, resulting in a worse progression free survival (PFS) and OS $(29,30)$. In addition to KRAS, TP53, CDKN2A/B and the MAPK/ERK pathway have been significantly correlated with a worse survival in CCA, with no OS difference with respect to the tumor 
location among iCCA, pCCA/dCCA and gallbladder (22). Genetic alterations in the chromatin modulating genes, $B A P 1$ and PBRM1 were correlated with bone metastases and worse survival in pCCA/dCCA (30).

In addition to genetic alterations, phenotypic biomarkers can help to predict outcome response of CCA patients undergoing potentially curative treatments, e.g., surgical resection. S100A4 (formerly known as fibroblast specific protein-1) is a small cytoskeleton-associated, calciumbinding protein involved in the regulation of a number of cellular processes including cell cycle progression and differentiation, normally expressed by macrophages and mesenchymal cells. S100A4 has been implicated in tumor metastases of several epithelial cancers, including colorectal and breast. Studies from our group in 86 patients undergoing liver resection for CCA, demonstrated that an enhanced expression of S100A4 in the nucleus of malignant cholangiocytes (present in $43 \%$ of cases), even if involving a small proportion of neoplastic bile ducts $(<30 \%)$, correlated with a worse OS and increased metastatisation following surgery, without relevant differences between iCCA and pCCA/dCCA (31). In a recent paper on 91 CCAs (24 iCCAs, 33 pCCAs and 34 dCCAs) analyzed by IHC for the expression of mismatch repair (MMR) genes (MLH1, PMS2, MSH2, MSH6) and mesothelin, patients with MMR deficiency $(22 \%)$ had a lower mesothelin expression and a worse OS, compared with patients with MMR proficiency and high mesothelin levels (14.5 vs. 30 months) (32). Since S100A4 and MMR deficiency can be druggable by paclitaxel and immunotherapies (see below), these studies have lent support to the notion that availability of biomarkers of early metastatic behavior may help to allocate CCA patients to their best treatment. Following these lines, we next discuss novel treatment approaches derived from conventional chemotherapy, targeted therapies, antiangiogenic therapies and immunotherapy.

\section{Chemotherapy}

The role of chemotherapy in the treatment of CCA has been evaluated in both adjuvant and metastatic setting. Given the strong invasiveness of the tumor, most patients with CCA already have locally advanced or metastatic disease at the time of diagnosis, and in those patients where surgical resection is feasible, the percentage of disease recurrence with local and distant metastases is nearly $60 \%$, mostly occurring within the first and second year from surgery (33). These data have encouraged studies to investigate if adjuvant therapy strategies may have a role in patients with resected CCA. In a meta-analysis including 20 studies assessing adjuvant approaches in CCA (chemotherapy, radiotherapy or chemoradiotherapy), Horgan et al. concluded for a clinical benefit of adjuvant therapies compared with surgery alone in patients with R1 margin status or node involvement at the resection (34). More recently, two randomized phase III trials investigating the efficacy of adjuvant chemotherapy in patients with resected biliary tract cancer, led to contradictory results. In the BILCAP trial, 447 patients undergoing radical resection for gallbladder cancer or CCA were randomized to receive eight cycle of capecitabine-based chemotherapy or placebo. In the experimental arm, the median OS was 53 months (95\% CI, 40-NR), significantly higher than in the observation arm (36 months) (95\% CI, 30-44), HR 0.75 (95\% CI, $0.58-0.97 ; \mathrm{P}=0.028$ ), thereby resulting in a $25 \%$ lower risk of death associated with capecitabine (35).

In contrast, the PRODIGE 12-ACCORD 18 phase III French trial, with 196 patients randomized within 3 months from R0 or R1 resection of biliary tract cancer to receive either GEMOX for 12 cycles or surveillance, failed to show a significant improvement in recurrence free survival (RFS) of treated patients (HR: 0.83; 95\% CI, 0.58-1.19; $\mathrm{P}=0.31$ ) (36).

In patients with metastatic disease, systemic chemotherapy is the only treatment having demonstrated improvement in OS and quality of life (QOL). Two randomized trials showed a clinical benefit from chemotherapy over the best supportive care (BSC) alone, though with some limitations regarding patient selection and tumor types. In fact, the first study included patients also with advanced pancreatic cancer, while the second enrolled patients with only gallbladder cancer and not CCA $(37,38)$. Several non-randomized phase II trials have been conducted in biliary tract cancer, but only few prospective studies have investigated first-line chemotherapy. In 2010, the multicentre $\mathrm{ABC}-02$ phase III trial determined what is currently established as the standard of care therapy in patients with advanced biliary tract cancers. In this seminal study, 410 patients including locally advanced or metastatic CCA (242 patients), with gallbladder (148 patients) or ampullary cancer (20 pts), underwent cisplatin $\left(25 \mathrm{mg} / \mathrm{m}^{2}\right)$ followed by gemcitabine $\left(1,000 \mathrm{mg} / \mathrm{m}^{2}\right)$ for eight cycles or gemcitabine alone $\left(1,000 \mathrm{mg} / \mathrm{m}^{2}\right)$ for six cycles. At a median follow-up of 8.2 months, the median OS was 11.7 months with combination therapy and 8.1 months in the gemcitabine only group (HR: 0.64; 95\% CI, 0.52-0.80; $\mathrm{P}<0.001)$, while the median PFS was 
8.0 months in the cisplatin plus gemcitabine group and 5.0 months in the gemcitabine group $(\mathrm{P}<0.001)$. Tumor control was obtained in $81.4 \%$ of patients receiving gemcitabine plus cisplatin, compared with $71.8 \%$ of gemcitabine as single agent $(\mathrm{P}=0.049)$. No differences in both rate of response and treatment effect were observed between CCAs and gallbladders cancers.

The combination was safe and adverse events were similar in the two groups, except for neutropenia that was higher in the gemcitabine plus cisplatin group (39). Similar results in terms of disease control rate (DCR), PFS and OS were reported in a Japanese randomized multicentric trial of 84 patients (40). Since then, the ABC-02 study remains the largest phase III randomized trial carried out in advanced biliary tract cancers.

Other chemotherapeutic agents such as oxaliplatin, irinotecan, 5-fluorouracil (5-FU) and the derivatives capecitabine and S-1 have demonstrated some efficacy in several phase II trials, but no more effective results than gemcitabine plus cisplatin combination have been demonstrated by randomized phase III trials.

In the last years, treatment algorithms in gastrointestinal oncology have been improved by the inclusion of triplet drug regimens $(41,42)$. On these bases, more intensive chemotherapy schedules have been evaluated for the treatment of CCA. A regimen with 5-FU added to gemcitabine and cisplatin (GFP) was tested in 21 patients in a phase II trial showing a promising anti-tumor activity supported by a median OS of 18.8 months and a median time to progression (TTP) of 13.4 months (43). In the KHBO 1002 phase II trial, 50 patients with advanced biliary tract cancer were treated with $\mathrm{S}-1$, an oral fluoropyrimidine prodrug, in addiction to gemcitabine and cisplatin obtaining a survival benefit of 16.1 months with limited toxicities (44). The combination of gemcitabine, cisplatin and nabpaclitaxel has been recently assessed in a phase II trial of 60 patients with metastatic or unresectable biliary tract cancer. Median PFS was 11.4 months (95\% CI, 6.1-16.1) and median OS was 19.2 months (95\% CI, 13.6-NA), with a 1 -year survival rate of $67.6 \%$. Among the 51 patients evaluated for response, DCR was $84.3 \%$ and response rate (RR) $39 \%$; of note, following chemotherapy 12 patients with unresectable disease became eligible to surgery, and one achieved complete therapeutic response (45). Randomized phase III trials are sorely needed to confirm the promising results obtained with triplet regimens.

Patients with a good performance status [0-1] failing a first-line with gemcitabine plus cisplatin combination therapy, would deserve a second-line chemotherapy (46). Unfortunately, no prospective trials of second-line therapy have been investigated so far and in this setting, the optimal treatment is ill-defined. A systematic review published in 2014 including phase II clinical trials, retrospective analyses and case reports showed only limited advantages for patients undergoing a second-line therapy, with a mean OS of 6.6 months and a mean PFS of 2.8 months (47). A phase III trial comparing a second line therapy of FOLFOX versus BSC in patients with advanced biliary tract cancer progressed to a first-line treatment is currently ongoing (NCT01926236).

\section{Target therapies}

Even once established the gemcitabine plus cisplatin combination as the current standard of care for firstline systemic therapy, the prognosis of patients with CCA has remained dismal. In recent years, the promising results obtained from target therapies in different tumor types, such as breast, lung, and colorectal cancer, and the identification by groundbreaking technologies such as NGS of novel putative oncogenic drivers eventually druggable also in CCA, have stimulated numerous clinical trials for the treatment of advanced and metastatic CCA (Table 2), though no target therapies are currently approved for CCA.

As previously discussed, FGFR2 fusions/translocations are present in $10-16 \%$ of iCCAs. FGFR inhibition is currently investigated in several clinical trials involving patients with CCA as performed for other solid advanced cancer types. Anti-FGFR strategies can be divided into non-selective and selective FGFR tyrosine kinase inhibitors (TKIs), FGFR ligand traps (small molecules able to bind and sequester FGFs, thus preventing their interaction with FRGR) and monoclonal antibodies. BGJ398, a pan-selective FGFR TKI, has shown anti-tumor activity in preclinical models of tumors harboring FGFR alterations. A phase II study has recently assessed BGJ398 effects in 61 patients affected by advanced or metastatic CCA containing FGFR2 fusions or other FGFR alterations with disease progression to previous therapy. The overall RR was $14.8 \%$ (18.8\% in FGFR2 fusions only), DCR was $75.4 \%$ (83.3\% FGFR2 fusions only), and estimated median PFS was 5.8 months (95\% CI, 4.3-7.6) (48). A number of anti-FGFR selective inhibitors, including Erdafitinib, Derazantinib, Ponatinib, TAS-120, INCB054828 and Debio 1347, are currently under investigation in CCAs and other advanced tumors harboring FGFR alterations (Table 2). 
Table 2 Current targeted therapy clinical trials in cholangiocarcinoma

\begin{tabular}{lcc}
\hline Target & Agent & NCT number \\
\hline FGFR & Derazantinib & NCT03230318 \\
FGFR & TAS 120 & NCT02052778 \\
FGFR & Debio 1347 & NCT01948297 \\
FGFR & INCB054828 & NCT02924376 \\
FGFR & Ponatinib & NCT02265341 \\
IDH1 & Ivosidenib & NCT02989857 \\
IDH1 & BAY 1436032 & NCT02746081 \\
IDH1, IDH2 & Olaparib & NCT03212274 \\
HER 2 & Trastuzumab & NCT02836847 \\
BRAF, MEK & Dabrafenib, trametinib & NCT02034110 \\
ALK/ROS1 & Ceritinib & NCT02374489 \\
ALK/ROS1 & Crizotinib & NCT02034981 \\
ROS1, ALK, TRKA, & Entrectinib & NCT02568267 \\
TRKB and TRKC & & NCT03093870 \\
EGFR, HER2, HER4 & Varlitinib & \\
\hline
\end{tabular}

Mutations of genes encoding for $I D H 1$ and $I D H 2$ $(m I D H 1, m I D H 2)$, are quite common genetic lesions in iCCA (25\%). Ivosidenib (AG-120) is a selective, reversible $\mathrm{mIDH} 1$ inhibitor that has been evaluated in a phase I trial of 72 mIDH1 CCA patients showing promising anti-oncogenic activity with a favourable safety profile: disease control was obtained in $56 \%$ of patients, whilst $6 \%$ experienced a partial response (PR) with a PFS at 6 months of $38.5 \%$ (49). A double-blind, placebo-controlled phase III randomized trial (ClarIDHy) of Ivosidenib is ongoing. BAY 1436032 is another oral mIDH1 inhibitor, whose safety, tolerability and preliminary anti-tumor activity are under evaluation in a phase I basket trial including $m I D H 1$ patients with advanced solid tumors including CCA. Other preclinical and clinical studies have investigated the possibility to target epigenetic mechanisms associated with CCA tumorigenesis and mediated by chromatin remodelling proteins (ARID1A, ARID1B, ARID2, BAP1, PBRM1, SMARCA2, SMARCA4 and SMARCAD1) using DNA methyltransferase inhibitors (DNMT), such as decitabine and azacitidine, and histone deacetylase inhibitors (HDAC), such as valproic acid, vorinostat and romidepsin (50-52). Among them, promising results have been obtained with valproic acid in a small phase I/II trial of 12 patients with advanced pancreatobiliary tract cancers, but further studies are necessary to evaluate the effectiveness of such approaches (53).

Along this line, recent studies highlighted a dependence of ovarian cancer bearing ARID1A inactivating mutations on HDAC6 activity (54). Since ARID1A mutations are also common in iCCA (55), clinical studies are needed to investigate whether HDAC6 inhibitors might represent a novel target therapy for ARID1A-mutated CCA.

The success of anti-EGFR inhibitors in the treatment of colorectal cancer and other malignancies has encouraged the assessment of these agents in CCA. In CCA, EGFR pathway activation is common in both iCCA and pCCA/ dCCA (about 20-30\%), and in vitro studies demonstrated that EGFR activation promotes the emergence of epithelial-to-mesenchymal (EMT)-like changes in cancer cells, supporting a more invasive cell phenotype (56). Consistent with these in vitro findings, EGFR blockade by its ATP-competitive inhibitor gefitinib reduced tumor dissemination in mice bearing subcutaneous CCA xenografts, an effect associated to a regression of EMT features in cancer cells $(57,58)$. In CCA, targeting EGFR is potentially a fascinating stratagem, since EGFR activation in CCA cells may rely either on autocrine (from the cancer cells themselves) (59) or paracrine (from the stromal microenvironment) (57) release of EGFR ligands. In a preliminary phase II trial, the chimeric EGFR antibody cetuximab was tested in addition to GEMOX in 30 patients with unresectable biliary tract cancer (27 CCAs) leading to objective responses in $63 \%$ of cases (39). However, the randomized phase II BINGO trial did not confirm the benefit derived from combining cetuximab to GEMOX in terms of PFS and OS (60). In contrast to colorectal cancer, the humanized EGFR antibody panitumumab added to GEMOX was not effective in patients with KRAS wildtype advanced biliary tract cancer (61). Erlotinib, an oral TKI of EGFR, has been studied in a phase II trial of 268 patients with previously untreated advanced biliary tract cancer (including gallbladder cancer) randomized to receive GEMOX with or without erlotinib (100 mg daily). The objective response rate was higher in the experimental arm (40 vs. 21 patients; $\mathrm{P}=0.005$ ), but no significant difference in PFS was noted between the two groups (HR: 0.80; 95\% CI, 0.61-1.03; P=0.087). Noteworthy, a subgroup analysis of CCA patients showed that addition of erlotinib to GEMOX significantly improved PFS [5.9 months (95\% CI, 4.7-7.1) for chemotherapy plus erlotinib vs 3.0 months (95\% CI, 1.1-4.9) for chemotherapy alone; HR: 0.73; 95\% CI, 0.53-1.00; $\mathrm{P}=0.049$ ] (62). More studies are necessary to understand if response to erlotinib in CCA correlates 
indeed with EGFR state mutation and to investigate the level of intra-tumor heterogenicity for EGFR alterations in CCA, which could be another factor limiting therapeutic activity of EGFR inhibitors. Only few data are available regarding effectiveness of anti-HER2 agents in CCA (63) and a phase II trial of trastuzumab plus GEMOX is still ongoing (NCT02836847). Varlitinib is an orally bioavailable inhibitor of EGFR, HER 2 and HER 4 currently assessed in a phase II trial in combination with capecitabine as a second-line systemic therapy (NCT03093870).

KRAS mutations are a common signature also in CCA (9-47\%) (30). This genetic alteration is druggable not by direct $K R A S$ inhibition but by targeting the downstream effectors $R A F$ and $M E K$. In a phase II study, the $M E K$ inhibitor selumetinib demonstrated anti-tumor activity in 28 patients with advanced biliary tract cancer, resulting in a median PFS of 3.7 months and a median OS of 9.8 months (64). BRAF mutations occur in only $3-5 \%$ of CCAs, predominantly iCCAs. In a phase II basket trial of Vemurafenib, an oral BRAF inhibitor, a PR was obtained in one out of 8 patients with CCA (65). A phase II trial of target therapy combining $B R A F$ and $M E K$ inhibitors is still ongoing (NCT02034110).

In iCCA, an integrative molecular analysis identified a "proliferating class" clustering in a significant proportion (62\%) with signatures of $R A S$ signaling activation, including mitogen-activated protein kinase and $M E T$, however, early phase clinical trials with the MET inhibitors cabozantinib and tivantinib showed only limited effects in CCAs $(66,67)$. Among MET antagonists, crizotinib and ceritinib are oral MET/anaplastic lymphoma kinase ALK/ROS inhibitors of a certain effectiveness in the treatment of NSCLC $(68,69)$. In a preclinical mouse allograft model of CCA, inactivation of FIG-ROS1 resulted in a significant inhibition of tumor growth (70). Two ongoing different phase II trials are assessing the efficacy of crizotinib and ceritinib in patients with advanced CCA (NCT02374489, NCT02034981) together with other advanced gastrointestinal malignancies. The phase II STARTRK-2 trial is evaluating entrectinib, a selective TKI of ROS1, ALK, TRKA, TRKB and TRKC, in patients harbouring ROS1 or ALK fusions (NCT02568267).

Poly ADP-ribose polymerase (PARP) inhibitors have demonstrated some anti-tumoral activity in different malignancies harboring somatic and/or germline mutations in the tumor-suppressor genes BRCA1 and BRCA2, and several clinical trials evaluating these compounds in patients affected by $B R C A$-mutated ovarian and breast cancers. In a retrospective study analysing 18 cases of $B R C A 1 / 2$-mutated
CCA from five centers, among four patients receiving PARP inhibitors against 13 treated with a platinumcontaining chemotherapy, one obtained sustained disease response with a PFS of 42.6 months. Since the prevalence of BRCA1/2 mutations in CCA is still uncertain, future studies of genomic profiling in larger cohorts will be necessary to address the therapeutic role of PARP inhibitors in this setting of patients (71). Sulkowski et al. have recently demonstrated in preclinical models that tumor cell sensitivity to PARP inhibitors can be enhanced by the oncometabolite 2-HG produced by neomorphic mIDH1/2 suppressing homologous recombination (72). Starting from these observations, a basket phase II trial of olaparib for patients with metastatic solid tumors with $I D H 1$ or IDH2 mutations including CCA has been undertaken (NCT03212274).

Focal losses of $C D K N 2 A / 2 B$ have been reported in nearly a half of iCCAs, and CDK4/6 inhibitors (palbociclib, ribociclib) have been successfully tested and recently approved in the treatment of breast cancer (73); however, anti-tumoral effects of these agents are yet to be determined in CCA.

Aberrant expression of S100A4 by neoplastic cells is an early feature of cancer-associated EMT (74), and in CCA, patients with nuclear expression of S100A4 in malignant cholangiocytes had a worse outcome, in terms of earlier metastasization and reduced survival after surgical resection (31). Importantly, nuclearization of S100A4 is not solely a prognostic biomarker, but also a mechanistic determinant of the motile and invasive properties of CCA cells, by activating the small Rho GTPases (RhoA and Cdc42), the secretion of active matrix metalloprotease (MMP)-9, and the expression of membrane-type (MT)1MMP (75). Data from our group also showed that in CCA cells, nuclear import of S100A4 is mediated by small ubiquitin-like modifier (SUMO)ylation, a post-translational modification of small proteins that are covalently attached to and detached from other proteins to critically regulate their intracellular localization, and this effect is halted by paclitaxel given at low doses. Starting from these in vitro findings, we tested the effects of metronomic infusion of paclitaxel in mice bearing orthotopic CCA xenografts. Noteworthy, inhibition of S100A4 nuclear entry by paclitaxel in engrafted cancer cells associated in vivo to a decreased hematogenous metastasization to the lungs without impinging tumor cell proliferation and/or apoptosis. Deregulation of SUMO-conjugating enzymes is a frequent event in cancer (76), where they 
likely stimulate plasticity of invasive carcinoma cells by targeting several EMT-inducing transcription factors (77). Overall, the 'S100A4 story' may be paradigmatic of how a tumoral biomarker with prognostic value can become target harnessed for therapeutic intervention.

\section{Angiogenesis inhibitors}

Since the early 2000's, angiogenesis inhibitors have been successfully utilized in the treatment of gastrointestinal and liver tumors, such as colorectal and gastric cancers, and hepatocellular carcinoma. At variance with hepatocarcinoma, CCA is characterized by a more abundant stromal reaction containing a limited number of blood vessels, in the face of a rich lymphatic vascularization, behaving as a preferential route of dissemination for cancer cells. Of note, lymph node metastasization is indeed an early event often ruling out indications to curative surgery, whereas distant metastases are not frequent (78). However, vascular endothelial growth factor (VEGF), a major proangiogenic factor involved in tumor's growth and metastatization, is overexpressed in $40-75 \%$ of biliary tract cancers, in particular at the front of tumoral invasion (17). A systematic analysis reported increased immunohistochemical expression levels of VEGF-A, the main isotype of the VEGF family encompassing five members from $-\mathrm{A}$ to $-\mathrm{D}$ and the placental growth factor, in iCCA compared with pCCA/ dCCA. This finding is consistent with the remarkable pro-angiogenic proclivity of the liver microenvironment, whereby many cell types beside tumoral cells, including also stromal and innate immune cells, cooperate to VEGF secretion (79). Antiangiogenic agents act through different mechanisms; some are monoclonal antibodies directed against VEGF, other are small molecules blocking VEGF signaling by binding VEGF or its cognate receptors (VEGFRs) or the downstream effectors.

Bevacizumab, a monoclonal antibody targeting VEGF-A, has been tested in combination with GEMOX in a phase II trial of 35 patients with advanced biliary tract cancer (including $25 \mathrm{CCAs}$ ), leading to a PR in $41 \%$ of patients, with a median PFS and OS of 7.6 and 14.2 months, respectively in the CCA subgroup (80). Bevacizumab was also evaluated in combination with erlotinib, a TKI of EGFR, mainly used in lung and pancreatic cancer. In a multinational phase II trial of 53 previously untreated patients with advanced biliary tract cancer (43 CCAs) bevacizumab combined with erlotinib showed promising results, 6 patients with a PR and 25 with a stable disease
(SD), with a median OS of 9.9 months and a TTP of 4.4 months (81).

Sorafenib, an oral panTKI (acting on VEGFR-2/3, PDGFR, BRAF and CRAF), has been evaluated in two different phase II trials. In the first, 46 patients $(400 \mathrm{mg}$ twice a day) showed a DCR at 12 weeks of $32.6 \%$, a PFS of 2.3 months and a medium OS of 4.4 months (82). The second study was closed because failing to meet the minimal criterion of at least one patient with confirmed PR; however, a stable disease with PFS of 3 months and OS of 9 months was obtained in $39 \%$ of cases (83). Addition of sorafenib or other TKIs to conventional chemotherapy agents did not result in any significant advantage in terms of DCR, PFS and OS with respect to the chemotherapy alone $(84,85)$.

Cediranib, an oral TKI acting on VEGFR1/2/3, PDGFR and c-Kit, was studied in a phase II trial of 124 patients with previously untreated advanced biliary tract cancer randomized to receive chemotherapy (cisplatin plus gemcitabine) with or without cediranib. No significant improvements in either PFS (8 months in the experimental arm and 7.4 months in control arm, HR: 0.93; 95\% CI, $0.65-1.35 ; \mathrm{P}=0.72)$ or OS (14.1 vs. 11.9 months; HR: 0.86; $\mathrm{P}=0.44)$ were observed. However, a significant correlation was observed between baseline levels of PDGF-BB (one of the targets of cediranib) and OS, meaning that patients with PDGF-BB levels above the median took benefit in terms of OS $(\mathrm{P}=0.002)$ (86).

Regorafenib is an oral multi-TKI enpowered with potent anti-angiogenic activity dependent on the triple blockade of VEGFRs, FGFRs and Tie2 (the cognate receptor for angiopoietins), approved for the treatment of metastatic colorectal cancer and advanced gastrointestinal tumours. Two different phase II studies on regorafenib in patients with advanced biliary tract cancer receiving no more than two prior lines of chemotherapy are currently ongoing (NCT02115542, NCT02053376).

Taken together, these data suggest that therapeutic antagonism of angiogenesis has only limited efficacy in the treatment of CCA, albeit sporadic studies have led to some promising results. Clinical trials on selected subgroups, identified by predictive biomarkers (as would be the case with PDGF-BB), are strongly needed to define setting of patients who might benefit more from anti-angiogenic agents.

\section{Immunotherapy}

Tumor evasion of immune surveillance is a crucial step in neoplastic progression. This dynamic process called 'cancer 
immunoediting' has been extensively studied in the last two decades leading to the recent concept that the immune system not only protects against cellular transformation, but also molds the immunogenicity of the developing tumors, i.e., the expression of tumor-specific antigens by the transformed cell itself (87).

Tumor cells are able to escape from immune surveillance adopting several strategies. These include: (I) loss or alteration of major histocompatibility complex (MHC) class 1 and other specific antigens, (II) up-regulation of immune check-point molecules such as programmed cell death protein 1 (PD-1) and T lymphocyte-associated antigen 4 (CTLA-4) that once activated by their specific ligands (PDL1 and CD152, respectively), promote peripheral $\mathrm{T}$ cell exhaustion, and (III) manipulation of the local immune microenvironment to become tolerant, by secreting cytokines suppressing proliferation of $\mathrm{CD}^{+}$and $\mathrm{CD}^{+} \mathrm{T}$ lymphocytes in charge of tumor antigen recognition or by recruiting myeloid-derived suppressor cells. Targeting these mechanisms, in particular interference with immune checkpoints (checkpoint inhibitors), has represented a major breakthrough in oncology research. Antibodies blocking the interaction of PD-1 and CTLA-4 with their specific ligands have been successful in the treatment of several hematological and solid malignancies, including Hodgkin's lymphoma, advanced melanoma and renal cell, colorectal or non-small cell lung carcinomas, thereby arising interest for immunotherapy also in CCA. Unravelling the molecular pathways regulating tumor cell interactions with the immune cells and finding specific predictive biomarkers of tumor response to immune checkpoint inhibitors are essential to identify patients that could take more advantage of immune-modulating therapies.

Several studies have reported that the total number of somatic mutations associated with a favourable clinical response to immune checkpoint inhibitors, as shown in melanoma, NSCLC and urothelial cancers $(88,89)$. Le et al. found that pembrolizumab, an anti-PD-1 antibody, was more effective against DNA MMR-deficient tumors than against DNA MMR-proficient tumors. Loss of DNA MMR results in a hypermutable phenotype called 'microsatellite instability' (MSI), featuring an exuberant generation of neoepitopes, which elicit a stronger immune response against tumor cells, as confirmed by prominent lymphocyte infiltrates around tumor cells and increased PD-L1 expression $(90,91)$. Based on these observations, the FDA has recently approved pembrolizumab for the treatment of adult and pediatric patients with unresectable or metastatic MSI-high or MMR-deficient solid tumors that have progressed after prior treatment and without alternative treatment options. In their seminal study, Nakamura et al. by whole exome sequencing identified a subgroup of CCA patients $(5.9 \%, 14 / 239)$ with poor prognosis characterized by high mutational load, abundant tumor-specific neoantigens and high expression of immune checkpoint molecules including PD-L1. They found that the $36 \%$ of these hypermutated tumors harbored inactivating mutations in the MMR complex (7). In a phase II trial (KEYNOTE-016), 86 patients with MMR-deficient tumors including 4 CCA were treated with pembrolizumab. Notably, preliminary data in CCA showed a $100 \%$ DCR with one complete response and three stable disease (92).

As aforementioned, an important biomarker predicting response to immunomodulators is the tumoral expression of PD-L1 as assessed by IHC (93-95). However, a crucial point is whether PD-L1 is expressed by tumoral cells, by infiltrating lymphocytes, or by both cell types. In a study evaluating PD-L1 expression in 99 cases of CCA (58 iCCA and 41 pCCA) by IHC, PD-L1 immunostaining was considered positive when a typical membranous pattern was present in at least $5 \%$ of tumor cells. While PDL1 expression confined to neoplastic cells was found in 9 cases, PD-L1 expression extended to inflammatory cell aggregates was detected in 46 cases, with dense intratumoral lymphocytic infiltration (96). Higher proportion of tumoral expression of PD-L1 CCAs (until 40\%) was reported by studies including cases with less than $5 \%$ of tumor cell staining (97). Although it is uncertain if PD-L1 expression by intratumoral microenvironment has the same predictive strength for treatment response as its expression by tumor cells, it is assumed that PD-L1 expression correlates to antitumor immunity suppression and $\mathrm{T}$ cell exhaustion (98). In a recent work, 91 CCA tissue specimens (24 iCCAs, 33 pCCAs and 34 dCCAs) were analyzed by IHC for MMR gene products (MLH1, PMS2, MSH2, MSH6), mesothelin, PD-L1 and immune cell infiltration. Interestingly, albeit MMR deficiency was found in 20 tumors (22\%), none of them co-expressed PD-L1 (set at $>1 \%$ of tumor cells); moreover, no differences in tumour lymphocyte infiltrate was noted between MMR-deficient or MMR-proficient tumors (32). In another recent German study performed in surgical specimens of CCA derived from resection, including 102 patients (35 iCCA, 42 pCCA, 25 dCCA), the prevalence of MSI was examined not only by IHC for the expression of MLH1, PMS2, MSH2, and MSH6, but also by pentaplex PCR for five quasimonomorphic 
mononucleotide repeats (BAT-25, BAT-26, NR-21, NR-22, and NR-24). Whereas in the IHC-based analysis, no loss of expression of DNA repair enzymes was observed, by PCR the authors found only $1 \%$ of MSI-high and $1 \%$ of MSI-low (99). Thus, in addition to highlight discrepancy between different techniques aimed at investigating MSI, this study argues against the actual relevance of MSI in CCA. However, it must be underlined that by analyzing patients undergoing a therapeutic approach with curative intent, the study might have selected a subgroup of CCA with a less aggressive phenotype.

Immune-modulating therapy trials have been recently planned for patients with advanced CCA characterized by dense intratumoral lymphocyte infiltration/PD-L1 expression. In the KEYNOTE-028 basket trial 24 patients with biliary tract cancer and PD-L1 expression $(\geq 1 \%$ of tumor cells by IHC) were treated with pembrolizumab with promising results: 4 patients (17\%) had PR and $4(17 \%)$ had stable disease (100). The KEYNOTE-158, a phase II basket trial of pembrolizumab in patients with advanced (unresectable or metastatic) solid tumors including CCAs progressed on standard of care therapy is currently ongoing (NCT02628067).

Altogether, immune checkpoint inhibitors seem to be effective in a subset of patients with advanced biliary tract expressing PD-L1 or with DNA MMR defects and/or MSIhigh. However, further studies are necessary to identify predictive biomarkers of response to these innovative therapeutic options.

\section{Conclusions}

In the last few years, the recent advances in the genetic understanding of many diseases, including cancer, have sped up the idea of a 'precision medicine'. A precision medicinebased approach aims at selecting the most appropriate treatment dependent on the specific molecular alterations displayed by the single patient, at odds with a standard, 'non-customized' approach, not rarely disappointing, offered to most patients once a disease is diagnosed and staged. In contrast with other 'luckier' cancer types, such as breast, lung and colorectal, the road towards a precision medicine in CCA is much more bumpy because of the wide heterogeneity of the tumor and the uncertainties on the genomic profile. Unfortunately, nodal molecular fingerprints addressing therapeutic decisions, as are RAS/ BRAF for colorectal cancer or EGFR for lung cancer, are currently lacking in CCA. Notwithstanding, some important nuances of molecular definition of CCA are beginning to emerge and the 'Rosetta stone' has started to be deciphered. Some gain in conventional chemotherapy has been obtained by the inclusion of triplet drug regimens, and randomized phase III trials are eagerly awaited to confirm the first promising results. Target therapies represent an area that more recent translational studies are strongly pushing forward. Among several molecular targets, FGFR, IDH, EGFR and S100A4 are frequently altered in CCA and they will deserve deep consideration for therapeutic intervention by future studies, including also adjuvant and/or neoadjuvant settings. Angiogenesis inhibitors have been disappointed so far, but conceivably because the major 'culprit' of CCA dissemination (lymphatic vascular system) has not been selectively targeted yet. Finally, immunotherapy might actually make the difference, but only once the real involvement of MSI and MMR deficiency in CCA has been better clarified. That said, further efforts are needed to improve the pipeline for developing new anticancer strategies in CCA. In this regard, development of additional groundbreaking technologies, such as the targeted capture sequencing to detect actionable alterations in formalin-fixed paraffin-embedded samples (101), or single-cell analysis with computational methods to characterize genome-scale molecular information at the individual cell level (102) will be valuable assets in the near future to bridge a gap that cannot wait any longer.

\section{Acknowledgements}

Funding: L Fabris is supported by the University of Padua, Progetti di Ricerca di Dipartimento (PRID) 2017; S Indraccolo is supported by Associazione Italiana Ricerca sul Cancro (AIRC), grant \#IG18803.

\section{Footnote}

Conflicts of Interest: The authors have no conflicts of interest to declare.

\section{References}

1. Brito AF, Abrantes AM, Encarnação JC, et al. Cholangiocarcinoma: from molecular biology to treatment. Med Oncol 2015;32:245

2. Banales JM, Cardinale V, Carpino G, et al. Expert consensus document: Cholangiocarcinoma: current knowledge and future perspectives consensus statement 
from the European Network for the Study of Cholangiocarcinoma (ENS-CCA). Nat Rev Gastroenterol Hepatol 2016;13:261-80.

3. Høgdall D, O'Rourke CJ, Taranta A, et al. Molecular Pathogenesis and Current Therapy in Intrahepatic Cholangiocarcinoma. Dig Dis 2016;34:440-51.

4. Loupakis F, Yang D, Yau L, et al. Primary tumor location as a prognostic factor in metastatic colorectal cancer. J Natl Cancer Inst 2015;107:dju427.

5. Missiaglia E, Jacobs B, D'Ario G, et al. Distal and proximal colon cancers differ in terms of molecular, pathological, and clinical features. Ann Oncol 2014;25:1995-2001.

6. Edge SB, Compton CC. The American Joint Committee on Cancer: the 7th edition of the AJCC cancer staging manual and the future of TNM. Ann Surg Oncol 2010;17:1471-4.

7. Nakamura H, Arai Y, Totoki Y, et al. Genomic spectra of biliary tract cancer. Nat Genet 2015;47:1003-1010.

8. Borad MJ, Gores GJ, Roberts LR. Fibroblast growth factor receptor 2 fusions as a target for treating cholangiocarcinoma. Curr Opin Gastroenterol 2015;31:264-268.

9. Borad MJ, Champion MD, Egan JB, et al. Integrated Genomic Characterization Reveals Novel, Therapeutically Relevant Drug Targets in FGFR and EGFR Pathways in Sporadic Intrahepatic Cholangiocarcinoma. PLoS Genet 2014;10:e1004135.

10. Sia D, Losic B, Moeini A, et al. Massive parallel sequencing uncovers actionable FGFR2-PPHLN1 fusion and ARAF mutations in intrahepatic cholangiocarcinoma. Nat Commun 2015;6:6087.

11. Gu TL, Deng X, Huang F, et al. Survey of tyrosine kinase signaling reveals ROS kinase fusions in human cholangiocarcinoma. PLoS One 2011;6:e15640.

12. Peraldo Neia C, Cavalloni G, Balsamo A, et al. Screening for the FIG-ROS1 fusion in biliary tract carcinomas by nested PCR. Genes Chromosom Cancer 2014;53:1033-40.

13. Jiao Y, Pawlik TM, Anders RA, et al. Exome sequencing identifies frequent inactivating mutations in BAP1, ARID1A and PBRM1 in intrahepatic cholangiocarcinomas. Nat Genet 2013;45:1470-3.

14. Mondesir J, Willekens C, Touat M, et al. IDH1 and IDH2 mutations as novel therapeutic targets: current perspectives. J Blood Med 2016;7:171-80.

15. Farshidfar F, Zheng S, Gingras MC, et al. Integrative Genomic Analysis of Cholangiocarcinoma Identifies Distinct IDH-Mutant Molecular Profiles. Cell Rep 2017;18:2780-94.

(C) Translational Gastroenterology and Hepatology. All rights reserved.
16. Nakazawa K, Dobashi Y, Suzuki S, et al. Amplification and overexpression of c-erbB-2, epidermal growth factor receptor, and c-met in biliary tract cancers. J Pathol 2005;206:356-65.

17. Yoshikawa D, Ojima H, Iwasaki M, et al. Clinicopathological and prognostic significance of EGFR, VEGF, and HER2 expression in cholangiocarcinoma. Br J Cancer 2008;98:418-25.

18. Leone F, Cavalloni G, Pignochino Y, et al. Somatic mutations of epidermal growth factor receptor in bile duct and gallbladder carcinoma. Clin Cancer Res 2006;12:1680-5.

19. Gwak GY, Yoon JH, Shin CM, et al. Detection of response-predicting mutations in the kinase domain of the epidermal growth factor receptor gene in cholangiocarcinomas. J Cancer Res Clin Oncol 2005;131:649-52.

20. Chang YT, Chang MC, Huang KW, et al. Clinicopathological and prognostic significances of EGFR, KRAS and BRAF mutations in biliary tract carcinomas in Taiwan. J Gastroenterol Hepatol 2014;29:1119-25.

21. Pignochino Y, Sarotto I, Peraldo-Neia C, et al. Targeting EGFR/HER2 pathways enhances the antiproliferative effect of gemcitabine in biliary tract and gallbladder carcinomas. BMC Cancer 2010;10:631.

22. Javle M, Bekaii-Saab T, Jain A, et al. Biliary cancer: Utility of next-generation sequencing for clinical management. Cancer 2016;122:3838-47.

23. Riener MO, Bawohl M, Clavien PA, et al. Rare PIK3CA hotspot mutations in carcinomas of the biliary tract. Genes Chromosom Cancer 2008;47:363-7.

24. Chan-On W, Nairismägi ML, Ong CK, et al. Exome sequencing identifies distinct mutational patterns in liver fluke-related and non-infection-related bile duct cancers. Nat Genet 2013;45:1474-8.

25. Jang S, Chun SM, Hong SM, et al. High throughput molecular profiling reveals differential mutation patterns in intrahepatic cholangiocarcinomas arising in chronic advanced liver diseases. Mod Pathol 2014;27:731-9.

26. Jusakul A, Cutcutache I, Yong CH, et al. Wholegenome and epigenomic landscapes of etiologically distinct subtypes of cholangiocarcinoma. Cancer Discov 2017;7:1116-35.

27. Farhat M-H. Prognostic factors in patients with advanced cholangiocarcinoma: Role of surgery, chemotherapy and body mass index. World J Gastroenterol 2008;14:3224.

28. Graham RP, Barr Fritcher EG, Pestova E, et al. Fibroblast growth factor receptor 2 translocations in intrahepatic 
cholangiocarcinoma. Hum Pathol 2014;45:1630-8.

29. Zhu AX, Borger DR, Kim Y, et al. Genomic Profiling of Intrahepatic Cholangiocarcinoma: Refining Prognosis and Identifying Therapeutic Targets. Ann Surg Oncol 2014;21:3827-34.

30. Churi CR, Shroff R, Wang Y, et al. Mutation profiling in cholangiocarcinoma: Prognostic and therapeutic implications. PLoS One 2014;9:e115383.

31. Fabris L, Cadamuro M, Moserle L, et al. Nuclear expression of S100A4 calcium-binding protein increases cholangiocarcinoma invasiveness and metastasization. Hepatology 2011;54:890-899.

32. Kunk PR, Obeid JM, Winters K, et al. Mismatch repair deficiency in cholangiocarcinoma. J Clin Oncol 2018;36:269.

33. Hasegawa S, Ikai I, Fujii H, et al. Surgical resection of hilar cholangiocarcinoma: Analysis of survival and postoperative complications. World J Surg 2007;31:1256-63.

34. Horgan AM, Amir E, Walter T, et al. Adjuvant therapy in the treatment of biliary tract cancer: A systematic review and meta-analysis. J Clin Oncol 2012;30:1934-40.

35. Primrose JN, Fox R, Palmer DH, et al. Adjuvant capecitabine for biliary tract cancer: The BILCAP randomized study. J Clin Oncol 2017;35:4006.

36. Edeline J, Bonnetain F, Phelip JM, et al. Gemox versus surveillance following surgery of localized biliary tract cancer: Results of the PRODIGE 12-ACCORD 18 (UNICANCER GI) phase III trial. J Clin Oncol 2017;35:225.

37. Glimelius B, Hoffman K, Sjödén PO, et al. Chemotherapy improves survival and quality of life in advanced pancreatic and biliary cancer. Ann Oncol. 1996;7:593-600.

38. Sharma A, Dwary AD, Mohanti BK, et al. Best supportive care compared with chemotherapy for unresectable gall bladder cancer: A randomized controlled study. J Clin Oncol 2010;28:4581-6.

39. Valle J, Wasan H, Palmer DH, et al. Cisplatin plus Gemcitabine versus Gemcitabine for Biliary Tract Cancer. N Engl J Med 2010;362:1273-81.

40. Okusaka T, Nakachi K, Fukutomi A, et al. Gemcitabine alone or in combination with cisplatin in patients with biliary tract cancer: A comparative multicentre study in Japan. Br J Cancer 2010;103:469-74.

41. Loupakis F, Cremolini C, Masi G, et al. Initial Therapy with FOLFOXIRI and Bevacizumab for Metastatic Colorectal Cancer. N Engl J Med 2014;371:1609-18.

42. Conroy T, Desseigne F, Ychou M, et al. FOLFIRINOX versus Gemcitabine for Metastatic Pancreatic Cancer. N Engl J Med 2011;364:1817-25.

43. Yamashita Y, Taketomi A, Itoh S, et al. Phase II trial of gemcitabine combined with 5 -fluorouracil and cisplatin (GFP) chemotherapy in patients with advanced biliary tree cancers. Jpn J Clin Oncol 2010;40:24-8.

44. Kanai M, Hatano E, Kobayashi S, et al. A multi-institution phase II study of gemcitabine/cisplatin/S-1 (GCS) combination chemotherapy for patients with advanced biliary tract cancer (KHBO 1002). Cancer Chemother Pharmacol 2015;75:293-300.

45. Shroff RT, Borad MJ, Xiao L, et al. A phase II trial of gemcitabine $(\mathrm{G})$, cisplatin $(\mathrm{C})$, and nab-paclitaxel $(\mathrm{N})$ in advanced biliary tract cancers (aBTCs). J Clin Oncol 2017;35:4018.

46. Fornaro L, Cereda S, Aprile G, et al. Multivariate prognostic factors analysis for second-line chemotherapy in advanced biliary tract cancer. Br J Cancer 2014;110:2165-9.

47. Lamarca A, Hubner RA, David Ryder W, et al. Secondline chemotherapy in advanced biliary cancer: a systematic review. Ann Oncol 2014;25:2328-2338.

48. Javle M, Lowery M, Shroff RT, et al. Phase II study of BGJ398 in patients with FGFR-Altered advanced cholangiocarcinoma. J Clin Oncol 2018;36:276-82.

49. Lowery MA, Abou-Alfa GK, Burris HA, et al. Phase I study of AG-120, an IDH1 mutant enzyme inhibitor: Results from the cholangiocarcinoma dose escalation and expansion cohorts. J Clin Oncol 2017;35:4015.

50. Baradari V, Höpfner M, Huether A, et al. Histone deacetylase inhibitor MS-275 alone or combined with bortezomib or sorafenib exhibits strong antiproliferative action in human cholangiocarcinoma cells. World J Gastroenterol 2007;13:4458-66.

51. Dawson MA, Kouzarides T. Cancer epigenetics: From mechanism to therapy. Cell 2012;150:12-27.

52. Kwak TW, Kim DH, Jeong Y, et al. Antitumor activity of vorinostat-incorporated nanoparticles against human cholangiocarcinoma cells. J Nanobiotechnology 2015;13:60.

53. Sriraksa R, Limpaiboon T. Histone deacetylases and their inhibitors as potential therapeutic drugs for cholangiocarcinoma - cell line findings. Asian Pac J Cancer Prev 2013;14:2503-8.

54. Bitler BG, Wu S, Park PH, et al. ARID1A-mutated ovarian cancers depend on HDAC6 activity. Nat Cell Biol 2017;19:962-73.

55. Simbolo M, Vicentini C, Ruzzenente A, et al. Genetic alterations analysis in prognostic stratified groups 
identified TP53 and ARID1A as poor clinical performance markers in intrahepatic cholangiocarcinoma. Sci Rep 2018;8:7119.

56. Lindsey S, Langhans SA. Epidermal growth factor signaling in transformed cells. Int Rev Cell Mol Biol 2015;314:1-41.

57. Clapéron A, Mergey M, Aoudjehane L, et al. Hepatic myofibroblasts promote the progression of human cholangiocarcinoma through activation of epidermal growth factor receptor. Hepatology 2013;58:2001-11.

58. Clapéron A, Mergey M, Nguyen Ho-Bouldoires TH, et al. EGF/EGFR axis contributes to the progression of cholangiocarcinoma through the induction of an epithelialmesenchymal transition. J Hepatol 2014;61:325-32.

59. Clapéron A, Guedj N, Mergey M, et al. Loss of EBP50 stimulates EGFR activity to induce EMT phenotypic features in biliary cancer cells. Oncogene 2012;31:1376-88.

60. Malka D, Cervera P, Foulon S, et al. Gemcitabine and oxaliplatin with or without cetuximab in advanced biliarytract cancer (BINGO): A randomised, open-label, noncomparative phase 2 trial. Lancet Oncol 2014;15:819-28.

61. Leone F, Marino D, Cereda S, et al. Panitumumab in combination with gemcitabine and oxaliplatin does not prolong survival in wild-type KRAS advanced biliary tract cancer: A randomized phase 2 trial (Vecti-BIL study). Cancer 2016;122:574-81.

62. Lee J, Park SH, Chang HM, et al. Gemcitabine and oxaliplatin with or without erlotinib in advanced biliarytract cancer: A multicentre, open-label, randomised, phase 3 study. Lancet Oncol 2012;13:181-8.

63. Law LY. Dramatic response to trastuzumab and paclitaxel in a patient with human epidermal growth factor receptor 2-positive metastatic cholangiocarcinoma. J Clin Oncol 2012;30:e271-3.

64. Bekaii-Saab T, Phelps MA, Li X, et al. Multi-institutional phase II study of selumetinib in patients with metastatic biliary cancers. J Clin Oncol 2011;29:2357-63.

65. Goeppert B, Frauenschuh L, Renner M, et al. BRAF V600E-specific immunohistochemistry reveals low mutation rates in biliary tract cancer and restriction to intrahepatic cholangiocarcinoma. Mod Pathol 2014;27:1028-34.

66. Goyal L, Zheng H, Yurgelun MB, et al. A phase 2 and biomarker study of cabozantinib in patients with advanced cholangiocarcinoma. Cancer 2017;123:1979-88.

67. Pant S, Saleh M, Bendell J, et al. A phase I dose escalation study of oral c-MET inhibitor tivantinib (ARQ 197) in combination with gemcitabine in patients with solid tumors. Ann Oncol 2014;25:1416-21.

68. Shaw AT, Kim TM, Crinò L, et al. Ceritinib versus chemotherapy in patients with ALK-rearranged nonsmall-cell lung cancer previously given chemotherapy and crizotinib (ASCEND-5): a randomised, controlled, openlabel, phase 3 trial. Lancet Oncol 2017;18:874-86.

69. Solomon BJ, Mok T, Kim D-W, et al. First-line crizotinib versus chemotherapy in ALK-positive lung cancer. $\mathrm{N}$ Engl J Med 2014;371:2167-77.

70. Saborowski A, Saborowski M, Davare MA, et al. Mouse model of intrahepatic cholangiocarcinoma validates FIGROS as a potent fusion oncogene and therapeutic target. Proc Natl Acad Sci 2013;110:19513-8.

71. Golan T, Raitses-Gurevich M, Kelley RK, et al. Overall Survival and Clinical Characteristics of BRCA-Associated Cholangiocarcinoma: A Multicenter Retrospective Study. Oncologist 2017;22:804-10.

72. Sulkowski PL, Corso CD, Robinson ND, et al. 2-Hydroxyglutarate produced by neomorphic IDH mutations suppresses homologous recombination and induces PARP inhibitor sensitivity. Sci Transl Med 2017;9:eaal2463.

73. Beaver JA, Amiri-Kordestani L, Charlab R, et al. FDA approval: Palbociclib for the treatment of postmenopausal patients with estrogen receptor-positive, HER2-negative metastatic breast cancer. Clin Cancer Res 2015;21:4760-6.

74. Boye K, Nesland JM, Sandstad B, et al. Nuclear S100A4 is a novel prognostic marker in colorectal cancer. Eur J Cancer 2010;46:2919-25.

75. Cadamuro M, Spagnuolo G, Sambado L, et al. Lowdose paclitaxel reduces S100A4 nuclear import to inhibit invasion and hematogenous metastasis of cholangiocarcinoma. Cancer Res 2016;76:4775-84.

76. Lee JS, Choi HJ, Baek SH. Sumoylation and its contribution to cancer. Adv Exp Med Biol 2017;963:283-98.

77. Bogachek MV, De Andrade JP, Weigel RJ. Regulation of epithelial-mesenchymal transition through sumoylation of transcription factors. Cancer Res 2015;75:11-5.

78. Cadamuro M, Stecca T, Brivio S, et al. The deleterious interplay between tumor epithelia and stroma in cholangiocarcinoma. Biochim Biophys Acta 2018;1864:1435-43.

79. Wiggers JK, Ruys AT, Groot Koerkamp B, et al. Differences in immunohistochemical biomarkers between intra- and extrahepatic cholangiocarcinoma: A systematic review and meta-analysis. J Gastroenterol Hepatol 2014;29:1582-94.

80. Zhu AX, Meyerhardt JA, Blaszkowsky LS, et al. Efficacy 
and safety of gemcitabine, oxaliplatin, and bevacizumab in advanced biliary-tract cancers and correlation of changes in 18-fluorodeoxyglucose PET with clinical outcome: a phase 2 study. Lancet Oncol 2010;11:48-54.

81. Lubner SJ, Mahoney MR, Kolesar JL, et al. Report of a multicenter phase II trial testing a combination of biweekly bevacizumab and daily erlotinib in patients with unresectable biliary cancer: A phase II consortium study. J Clin Oncol 2010;28:3491-7.

82. Bengala C, Bertolini F, Malavasi N, et al. Sorafenib in patients with advanced biliary tract carcinoma: A phase II trial. Br J Cancer 2010;102:68-72.

83. El-Khoueiry AB, Rankin CJ, Ben-Josef E, et al. SWOG 0514: a phase II study of sorafenib in patients with unresectable or metastatic gallbladder carcinoma and cholangiocarcinoma. Invest New Drugs 2012;30:1646-51.

84. Lee JK, Capanu M, O'Reilly EM, et al. A phase II study of gemcitabine and cisplatin plus sorafenib in patients with advanced biliary adenocarcinomas. Br J Cancer 2013;109:915-9.

85. Moehler M, Maderer A, Schimanski C, et al. Gemcitabine plus sorafenib versus gemcitabine alone in advanced biliary tract cancer: A double-blind placebo-controlled multicentre phase II AIO study with biomarker and serum programme. Eur J Cancer 2014;50:3125-35.

86. Valle JW, Wasan H, Lopes A, Backen AC, et al. Cediranib or placebo in combination with cisplatin and gemcitabine chemotherapy for patients with advanced biliary tract cancer (ABC-03): A randomised phase 2 trial. Lancet Oncol 2015;16:967-78.

87. Mittal D, Gubin MM, Schreiber RD, et al. New insights into cancer immunoediting and its three component phases-elimination, equilibrium and escape. Curr Opin Immunol 2014;27:16-25.

88. Snyder A, Makarov V, Merghoub T, et al. Genetic Basis for Clinical Response to CTLA-4 Blockade in Melanoma. N Engl J Med 2014;371:2189-99.

89. Rosenberg JE, Hoffman-Censits J, Powles T, et al. Atezolizumab in patients with locally advanced and metastatic urothelial carcinoma who have progressed following treatment with platinum-based chemotherapy: A single-arm, multicentre, phase 2 trial. Lancet 2016;387:1909-20.

90. Dudley JC, Lin MT, Le DT, et al. Microsatellite instability as a biomarker for PD-1 blockade. Clin Cancer Res 2016;22:813-20.

91. Le DT, Uram JN, Wang H, et al. PD-1 Blockade in Tumors with Mismatch-Repair Deficiency. N Engl J Med
2015;372:2509-20.

92. Le DT, Durham JN, Smith KN, et al. Mismatch repair deficiency predicts response of solid tumors to PD-1 blockade. Science 2017;357:409-13.

93. Taube JM, Klein A, Brahmer JR, et al. Association of PD1, PD-1 ligands, and other features of the tumor immune microenvironment with response to anti-PD-1 therapy. Clin Cancer Res 2014;20:5064-74.

94. Herbst RS, Soria JC, Kowanetz M, et al. Predictive correlates of response to the anti-PD-L1 antibody MPDL3280A in cancer patients. Nature 2014;515:563-7.

95. Meng X, Huang Z, Teng F, et al. Predictive biomarkers in PD-1/PD-L1 checkpoint blockade immunotherapy. Cancer Treat Rev 2015;41:868-76.

96. Fontugne J, Augustin J, Pujals A, et al. PD-L1 expression in perihilar and intrahepatic cholangiocarcinoma. Oncotarget 2017;8:24644-51.

97. Gandini S, Massi D, Mandala M. PD-L1 expression in cancer patients receiving anti PD-1/PD-L1 antibodies: A systematic review and meta-analysis. Crit Rev Oncol Hematol 2016;100:88-98.

98. Ohaegbulam KC, Assal A, Lazar-Molnar E, et al. Human cancer immunotherapy with antibodies to the PD-1 and PD-L1 pathway. Trends Mol Med 2015;21:24-33.

99. Winkelmann R, Schneider M, Hartmann S, et al. Microsatellite Instability Occurs Rarely in Patients with Cholangiocarcinoma: A Retrospective Study from a German Tertiary Care Hospital. Int J Mol Sci 2018;19:E1421.

100. O'Neil BH, Wallmark J, Lorente D, et al. Pembrolizumab (MK-3475) for patients (pts) with advanced colorectal carcinoma (CRC): Preliminary results from KEYNOTE-028. Eur J Cancer 2015;51:S103.

101. Moorcraft SY, Gonzalez de Castro D, Cunningham D, et al. Investigating the feasibility of tumour molecular profiling in gastrointestinal malignancies in routine clinical practice. Ann Oncol 2018;29:230-6.

102. Yuan GC, Cai L, Elowitz M, et al. Challenges and emerging directions in single-cell analysis. Genome Biol 2017;18:84.

doi: $10.21037 / \operatorname{tgh} .2018 .07 .02$

Cite this article as: Pellino A, Loupakis F, Cadamuro M, Dadduzio V, Fassan M, Guido M, Cillo U, Indraccolo S, Fabris L. Precision medicine in cholangiocarcinoma. Transl Gastroenterol Hepatol 2018;3:40. 